\title{
Dystrophic Epidermolysis Bullosa Pruriginosa Presenting with Flagellate Scarring Pattern Before and After Treatment
}

\author{
Safia T. Metwaly, Zizette A. Moussa, Nivin Y. K. Shenouda, Mounira Waked, Mariam Alfons, Ghada \\ M. Raslan, Ahmed Sadek, Fady W. Georgy
}

\author{
*Al-Haud AL-Marsoud Hospital for Dermatology, Venereology and Andrology, Cairo, Egypt \\ *Corresponding Author: Fady Waheed Shawkey Georgy, E-mail: fadywaheed.fw@gmail.com
}

\begin{abstract}
Introduction: Dystrophic Epidermolysis Bullosa Pruriginosa (DEB-Pr) is a rare subtype of DEB which is characterized by intense pruritus and leading to skin blistering and nodular or lichenoid lesions most prominent on the extremities, especially shins of tibia. Toenail dystrophy is also frequently observed, but not necessary for diagnosis. Although many cases of DEB-Pr are caused by mutations of type VII collagen due to reported variants in COL7A1 gene, the clinical pictures vary. We report a patient with DEB-Pr who presented with striking flagellate hypertrophic scarring lesions. Histopathological and electron microscopic findings are essential for diagnosis, given the considerable overlap with clinically similar presentations such as hypertrophic lichen planus, prurigo nodularis, lichen simplex chronicus and dermatitis artefacta. Case Presentation: Herein, we report a case with a history of blistering since childhood followed by intensely pruritic papulonodules, predominantly on the shins of tibia and dorsum of feet with distinct flagellate scarring pattern and toenail dystrophy. Our case was monitored before and after treatment with topical Tacrolimus $0.03 \%$ ointment and systemic Cyclosporine. Conclusion: Our case had an excellent response to topical Tacrolimus $0.03 \%$ ointment and systemic Cyclosporine treatment.
\end{abstract}

Keywords: Dystrophic Epidermolysis Bullosa Pruriginosa, Pretibial Epidermolysis Bullosa, Histopathology, Genodermatoses, Mechanobullous.

\section{INTRODUCTION}

Epidermolysis Bullosa (EB) is a mechanobullous genodermatosis characterized by skin fragility and trauma-induced blisters. Dystrophic Epidermolysis Bullosa (DEB) is a clinically heterogeneous subgroup of EB caused by mutations in COL7AI (OMIM 120120), which encoded type VII collagen, gave rise to defective anchoring fibrils at the dermoepidermal junction, as well as sublamina densa blistering as a consequence ${ }^{[1]}$. DEB-Pr is an unusual clinical subtype of DEB characterized by severe pruritus, resulting in scratching-induced secondary linear pruriginous or lichenified plaques and marked scarring, especially on the shins of tibia ${ }^{[2]}$. Most cases were sporadic ${ }^{[3]}$. However, a few showed autosomal dominant or autosomal recessive pattern of inheritance [2, 4]. Common clinical differential diagnoses included hypertrophic lichen planus, prurigo nodularis, lichen simplex chronicus, lichen amyloidosis, keratosis lichenoides chronica (Nekam's Disease) and dermatitis artefacta ${ }^{[2,5,6]}$.

\section{CASE REPORT:}

A 48-year-old male carpenter was presented with flagellate scars and bullae on shins of tibia and papulo-nodules on thighs, forearms, back, buttocks and thighs with toenail dystrophy [Figure 1a \& $\mathbf{1 b}$ ]. He gave a history of having mild to moderate traumainduced skin blistering during childhood and teenage years. 5 years ago, he reported his conditioned worsened with severe pruritus leading to the aforementioned lesions. There was no history of drug intake or seasonal variation or exacerbation before the onset of his lesions. The patient was not diabetic nor hypertensive nor on any form of medication. He had no known allergies to drugs or food.

There was no history of consanguinity. $\mathrm{He}$ gave a positive family history; his late mother was suffered from the same dermatologic condition, as he was 18 years old son. His late mother was suffered from toenail dystrophy. The son also had toenail dystrophy; however, he was just starting to demonstrate mild to moderate trauma-induced skin blisters leaving post-inflammatory scars and hyperpigmentation like his father used to in his childhood and teenage years [Figure 2].

By examination, our patient had multiple, excoriated, hypertrophic, violaceous, linear papules, plaques, nodules as well as bullae forming distinct flagellate patterns on shins of tibia and the dorsum of his feet. His lesions were also found on forearms, back, buttocks and thighs. [Figures 1a]

Histopathology revealed a sub epidermal blister with hyperkeratosis [Figure 3]. Transmission 
electron microscope revealed dermo-epidermal junction separation at sub Lamina Densa [Figure 4].

On admission, the patient weighed 75 kilograms. His hematological parameters, blood pressure and renal functions were normal. He was then started on topical Tacrolimus $0.03 \%$ ointment as well as systemic Cyclosporine $(2 \mathrm{mg} / \mathrm{kg} / \mathrm{day})$ until his condition was stabilized and was discharged a week later. In total, we had our patient on a four-week regimen of Tacrolimus $0.03 \%$ ointment and Cyclosporine ( $2 \mathrm{mg} / \mathrm{kg} / \mathrm{day})$ for the first three weeks, then his dose was tapered to Cyclosporine $100 \mathrm{mg}$ during the fourth week of therapy. Our patient's renal functions as well as hematological parameters were monitored weekly and his blood pressure daily whilst on Cyclosporine. Within the first week of therapy the severe itching disappeared and the blister formation decreased immensely. After this four-week regimen, our patient's lesions were flattened; the itching stopped and his blistering ceased completely [Figure 1c \& 1d]. Currently, our patient was on a maintenance dose of Cyclosporine $50 \mathrm{mg}$ with successful symptomatic control.
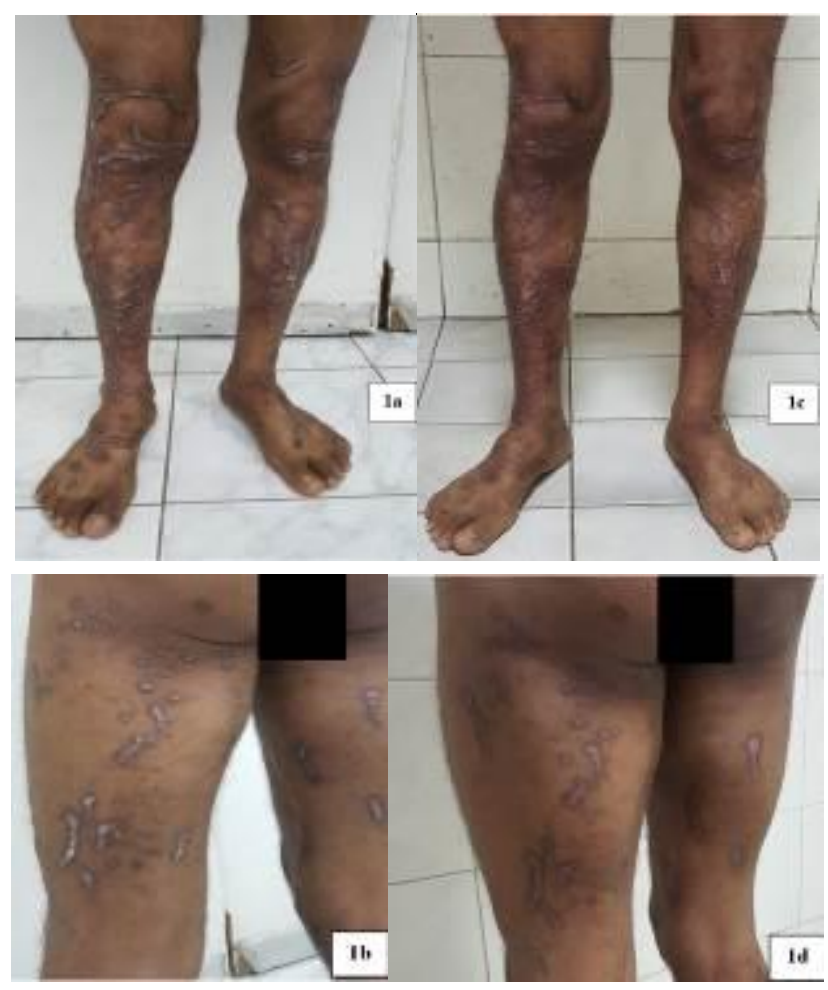

Figure 1. Clinical features of the flagellate pattern on shins of tibia, toenail dystrophy and thighs a $\&$ b before and $\mathrm{c} \& \mathrm{~d}$ after a four-week regimen of topical Tacrolimus $0.03 \%$ ointment and Cyclosporine.

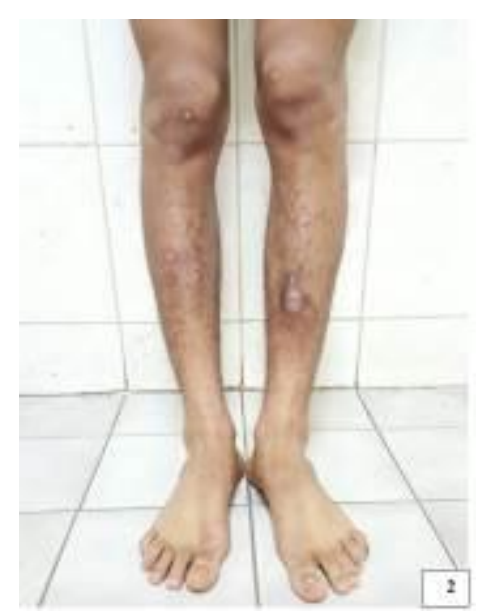

Figure 2: Clinical features of our patient's 18-year-old son showing erosions and blisters. Healing with post inflammatory hyper-pigmentation and scarring on his shins of tibia in addition to toenail dystrophy.

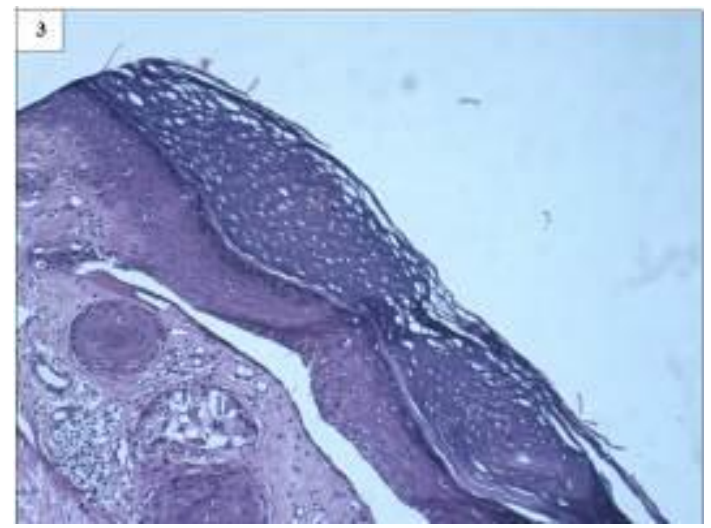

Figure 3. Histopathology shows a sub epidermal blister with hyperkeratosis.

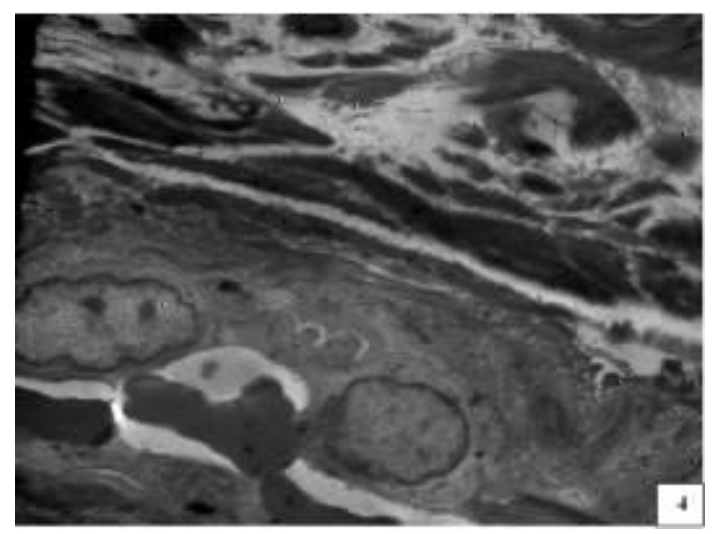

Figure 4. Transmission Electron Microscope shows separation at sub Lamina Densa.

\section{DISCUSSION}

Dystrophic Epidermolysis Bullosa Pruriginosa is a type of dystrophic EB coined and termed by McGrath et al. ${ }^{[2]}$. In the one original series of the eight cases he reported, three had family history of 
similar skin disease, two showing an autosomal dominant mode of inheritance, while the other an autosomal recessive pattern of inheritance.

Even though the pathogenesis is unknown; some authors demonstrated results with topical Tacrolimus $0.03 \%$ therapy alone ${ }^{[7]}$. Others have shown good results with systemic Thalidomide, while some have shown excellent results with systemic Cyclosporine ${ }^{[8-10]}$. Treatment was symptomatic and aimed to control pruritus and the progression of cutaneous lesions ${ }^{[11]}$. Potent topical steroids and intralesional triamcinolone were successful in some cases. Systemic antihistamines as well as corticosteroids had no good effect ${ }^{[7,8]}$.

Bazargan et al. ${ }^{[10]}$ postulated that elevated IgE in serum can cause pruritus in DEB-Pr. That is why they used Cyclosporine to eradicate pruritus by decreasing serum IgE levels ${ }^{[10]}$. Such results prompted us to have our patient on systemic Cyclosporine which has been very beneficial in treating our patient. We performed a literature search for cases presenting with linear scarring lesions and found only five cases with flagellate lesions similar to our own ${ }^{[12-15]}$. Morimoto et al. ${ }^{[15]}$ attributed the flagellate lesions in their patient to recurrent itching, probably due to the DEB-Pr itself, thus the tissue modification occurred as a result of such scratching.

\section{CONFLICTS of INTEREST}

There were no conflicts of interest.

\section{REFERENCES}

1. Fine JD, Eady RA, Bauer EA, Bauer JW, Bruckner-Tuderman $L$ et al. (2008): The classification of inherited epidermolysis bullosa (EB. J. Am. Acad.Dermatol., 58(6):931-950.

2. McGrath JA, Schofield OM and Eady RA (1994): Epidermolysis bullosa pruriginosa: dystrophic epidermolysis bullosa with distinctive clinicopathological features. Br. J. Dermatol., 130(5):617-625.

3. Wojnarowska F et al. (1998): Rook's Textbook of Dermatology. Blackwell Science. Oxford. pp: 1817-97.

4. Fine JD et al.(2010): Rook's Textbook of Dermatology. $8^{\text {th }}$ ed. Blackwell Science. Oxford. p p: 22-39.
5. Tey HL, Lee AD, Almaani N, McGrath JA, Mills KC and Yosipovitch G (2011): Epidermolysis bullosa pruriginosa masquerading as psychogenic pruritus. Arch Dermatol., 147(8):956-960.

6. Goulden V, Handfield-Jones S, Neild V and Black MM (1993): Linear prurigo simulating dermatitis artefacta in dominant dystrophic epidermolysis bullosa. Br. J. Dermatol., 129(4):443-446.

7. Banky JP, Sheridan AT, Storer EL and Marshman G (2004): Successful treatment of epidermolysis bullosa pruriginosa with topical tacrolimus. Arch Dermatol., 140:794-800.

8. Ozanic Bulic S, Fassihi H, Mellerio JE, McGrath JA and Atherton DJ (2005): Thalidomide in the management of epidermolysis bullosa pruriginosa. Br. J. Dermatol., 152(6):1332-1336.

9. Takahashi T, Mizutani Y, Ito M, Nakano H, Sawamura D and Seishima M (2016): Dystrophic epidermolysis bullosa pruriginosa successfully treated with immunosuppressants.J.Dermatol., 43(11):1391-1394.

10. Bazargan AS, Abdollahimajd F, Toosi $P$ and Azizian $Z$ (2017): Successful Treatment of Epidermolysis Bullosa Pruriginosa with Oral Cyclosporine. J. Skin Stem Cell, 4(3-4):74254-74259.

11. Pai V, Rai V, Athanikar S, Naveen $K$, Sori $T$ and Shastry $D$ (2014): Epidermolysis bullosa pruriginosa: A report of two cases. Indian Dermatology Online Journal, 5(1): 44-55.

12. Brick K, Hand JL, Frankel AS, Siegel, DH, Thomas KB, el-Azhary $R$ and Krol A (2012): Epidermolysis bullosa pruriginosa: further clarification of the phenotype. Pediatr. Dermatol., 29: 732-739.

13. Ee HL, Liu L, Goh CL and McGrath JA (2007): Clinical and molecular dilemmas in the diagnosis of familial epidermolysis bullosa pruriginosa. J. Am. Acad. Dermatol., 56: 77-81. 
14. Lee JY, Pulkkinen L, Liu HS Chen YF and Uitto J (1997): A glycine-to-arginine substitution in the triple-helical domain of type VII collagen in a family with dominant dystrophic epidermolysis bullosa pruriginosa. J Invest Dermatol., 108: 947-9.
15. Morimoto N, Shimizu A, Hattori M, Kuriyama Y, Nakano $H$ and Ohnishi $K$ (2018): Dystrophic epidermolysis bullosa pruriginosa presenting with flagellate scarring lesions. Clin Exp Dermatol., Oct 4. 